\title{
August 2013 Imaging Case of the Month
}

Michael B. Gotway, M.D.

\author{
Department of Radiology \\ Mayo Clinic Arizona \\ Scottsdale, AZ
}

\section{Clinical History}

A 60-year-old man presented with gradually worsening shortness of breath and dry cough over the previous six months, unresponsive to presumptive antibiotic therapy with levofloxacin first, followed by azithromycin. Frontal chest radiography (Figure 1) was performed.

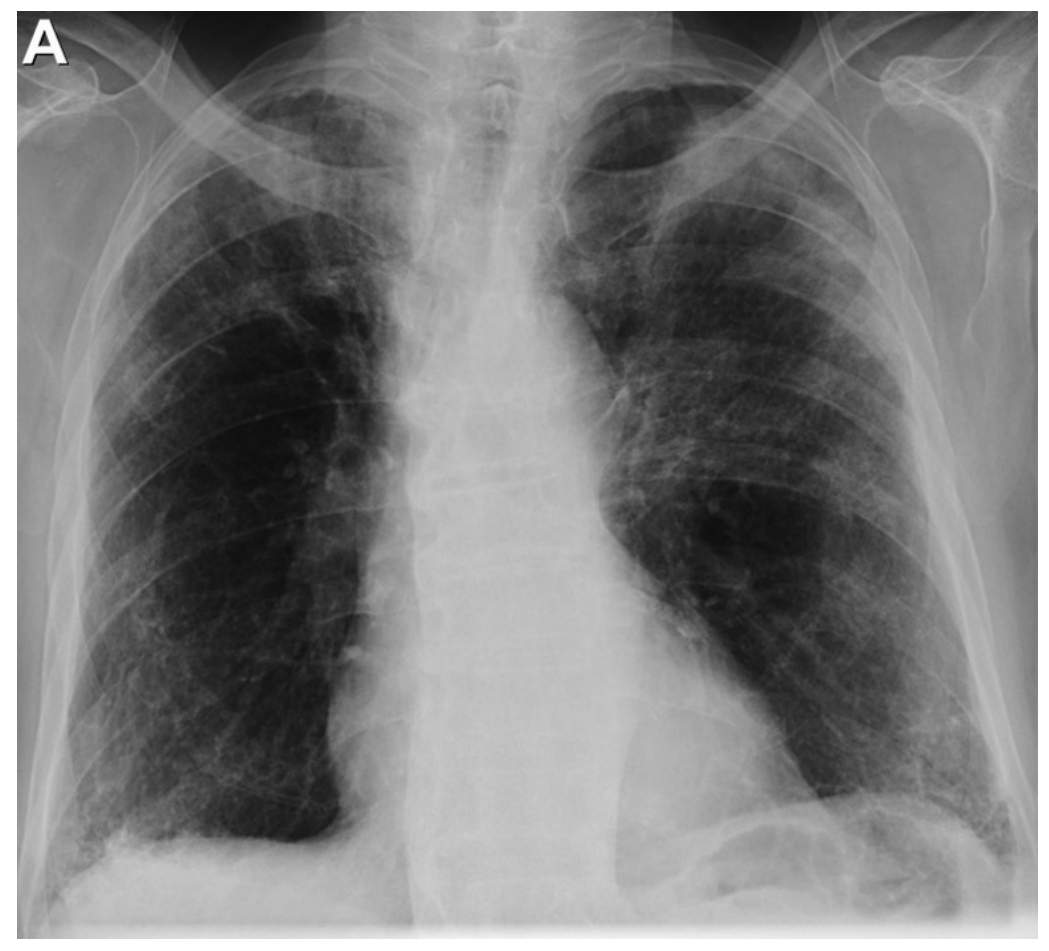

Figure 1. Frontal chest radiography.

Which of the following statements regarding the chest radiograph is most accurate?

1. The chest radiograph shows basal predominant fibrotic lung disease

2. The chest radiograph shows large lung volumes with cystic change

3. The chest radiograph shows multifocal bronchiectasis

4. The chest radiograph shows multiple nodules

5. The chest radiograph shows upper lobe peripheral consolidation and reticulation 


\section{Correct! \\ 5. The chest radiograph shows upper lobe peripheral consolidation and reticulation}

The frontal chest radiograph shows upper lobe, peripherally predominant opacities consisting of consolidation, best seen in the subpleural left upper lobe, and underlying linear and reticular opacities. These findings are not basal predominant. No nodules are present. Some areas of airway thickening are difficult to exclude but clear evidence of bronchiectasis is lacking. The lung volumes are normal to increased, although the diaphragms are not flattened, but no cystic change is present.

Which of the following is an appropriate consideration among the differential diagnostic possibilities for the appearance of the patient's chest radiograph?

1. Aspiration pneumonia

2. Bilateral bronchopneumonia

3. Chronic eosinophilic pneumonia

4. Idiopathic interstitial pneumonia

5. All of the above 


\section{Correct! \\ 5. All of the above}

Any of the above entities could present with peripheral opacities on chest radiography. Bronchopneumonia is not typically peripherally predominant, but it is such a common clinical entity that an uncommon, or even frankly rare, manifestation will occasionally be encountered. Several of the idiopathic interstitial pneumonias may present with peripheral opacities. Usual interstitial pneumonia / idiopathic pulmonary fibrosis commonly presents with peripheral or frankly subpleural opacities, although such opacities are usually basal predominant; the same is true of non-specific interstitial pneumonia. Organizing pneumonia, either cryptogenic or secondary, classically presents with peripheral or frankly subpleural opacities, often consolidation, on chest radiography. Chronic eosinophilic pneumonia also characteristically presents with peripheral opacities on chest radiography and thoracic CT. Aspiration also frequently involves the peripheral and subpleural lungs, although the peripheral location is often noted in the context of a dependent distribution as well.

The patient underwent routine thoracic CT (Figure 2) for further characterization of the abnormalities seen at chest radiography.

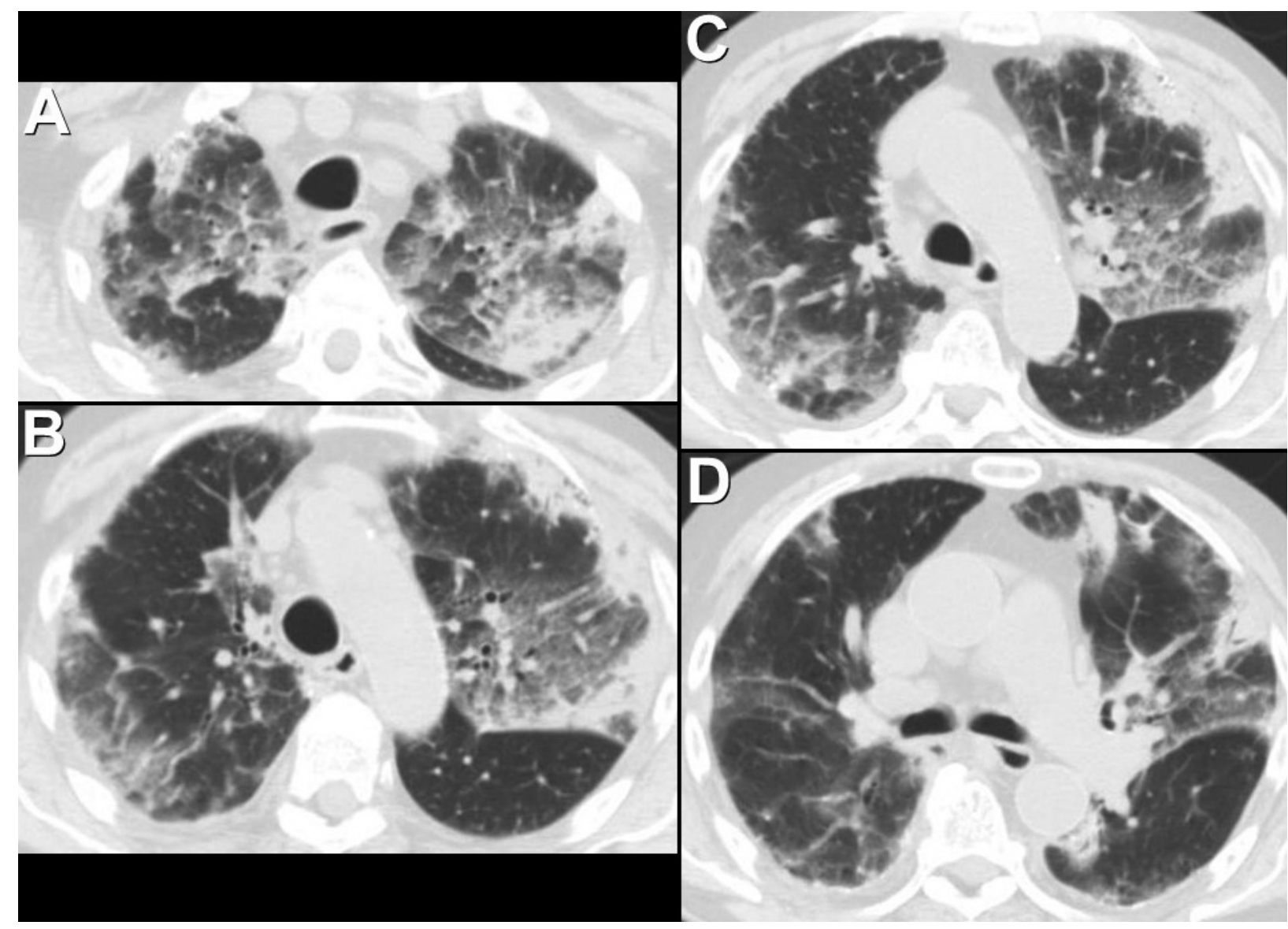

Figure 2. Panels A-D. Representative static images from routine thoracic CT scan. 
An axial high-resolution thoracic CT (E-J) was also performed (Figure 2, Panels E-J).

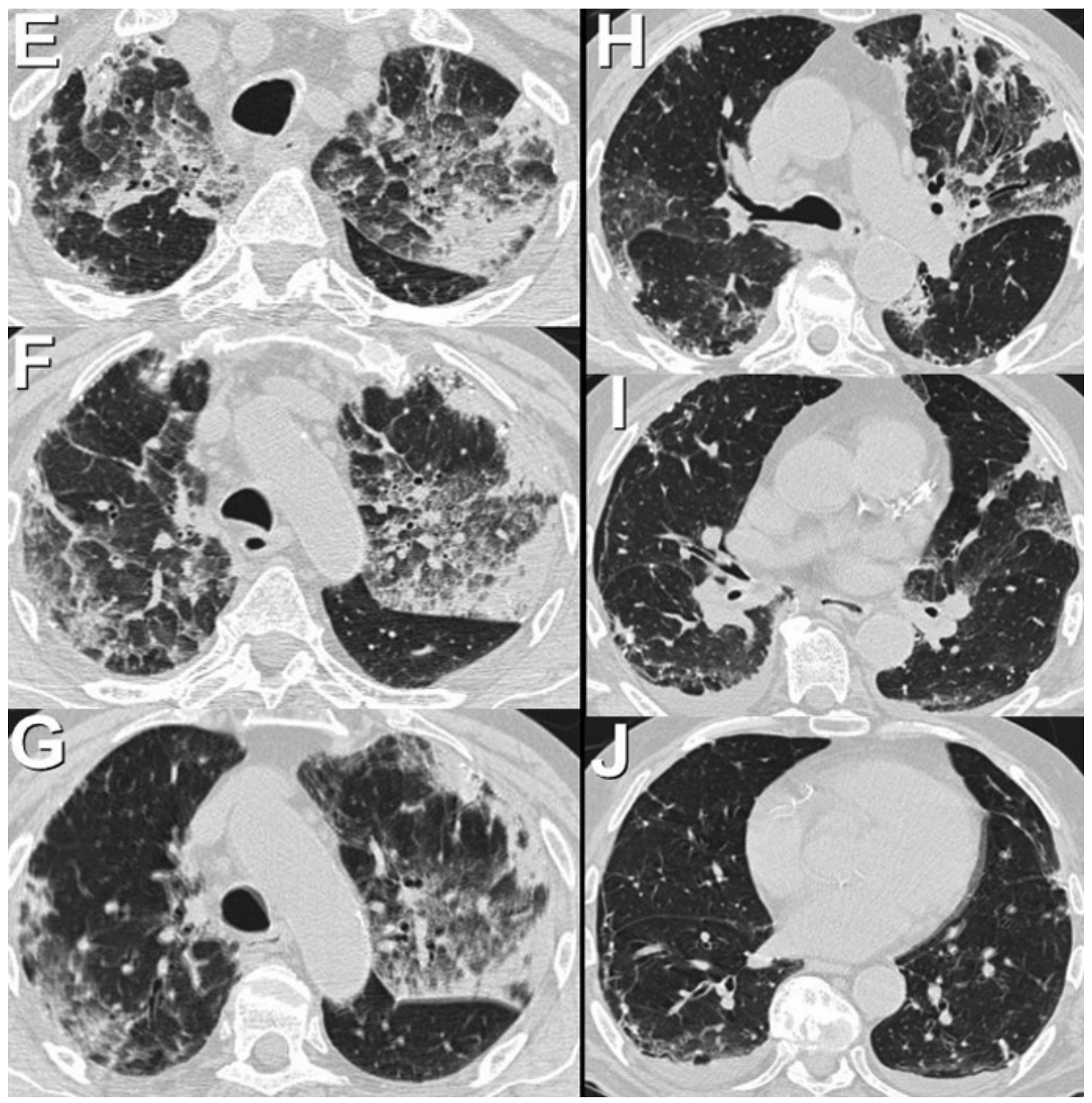

Figure 2. Panels E-J. Selected static axial images from the high-resolution thoracic CT scan.

Which of the following statements regarding this CT examination is most accurate?

1. The thoracic CT shows multifocal ground-glass opacity and consolidation, some of which is peripheral

2. The thoracic CT shows multifocal ground-glass opacity nodules

3. The thoracic CT shows peribronchovascular nodular interstitial thickening

4. The thoracic CT shows peripheral endobronchial airway impaction

5. The thoracic CT shows that the opacity on the chest radiograph is cavitary

\section{Correct!}




\section{The thoracic CT shows multifocal ground-glass opacity and consolidation, some of which is peripheral}

The thoracic CT shows peripheral consolidation- areas of increased lung attenuation that obscures the margins of vessels and bronchi. Some of the opacities are groundglass in attenuation also- areas of increased lung attenuation that does not obscure the margins of vessels and bronchi. Many of the opacities are frankly subpleural-so peripherally positions that the form a band-like opacity paralleling the chest wall immediately adjacent to the visceral pleural surface (Figure 3).

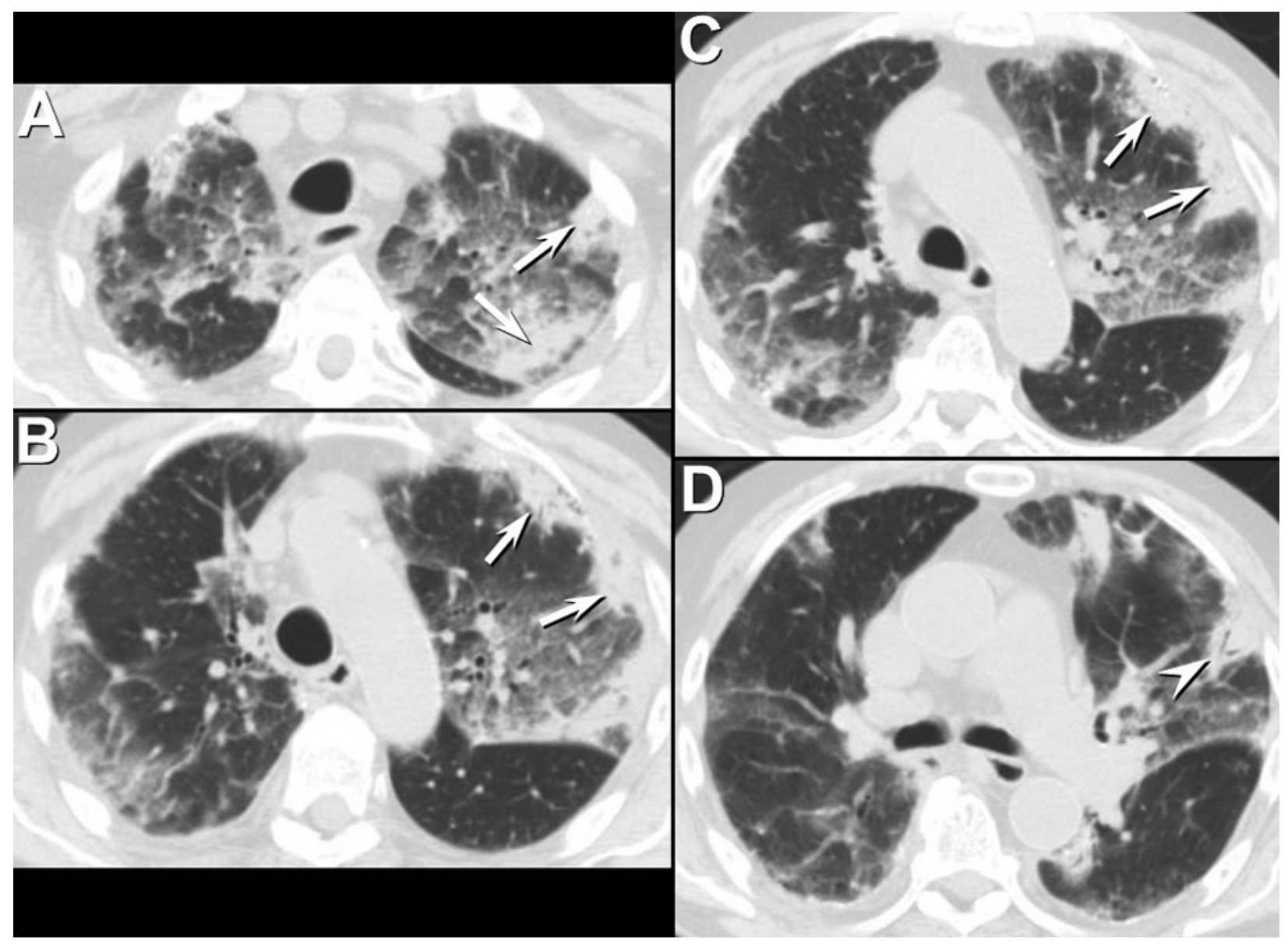

Figure 3. Panels A-D. Routine axial thoracic CT displayed in lung windows shows patchy areas of ground-glass opacity and consolidation (arrows) which is noticeably peripherally distributed. 


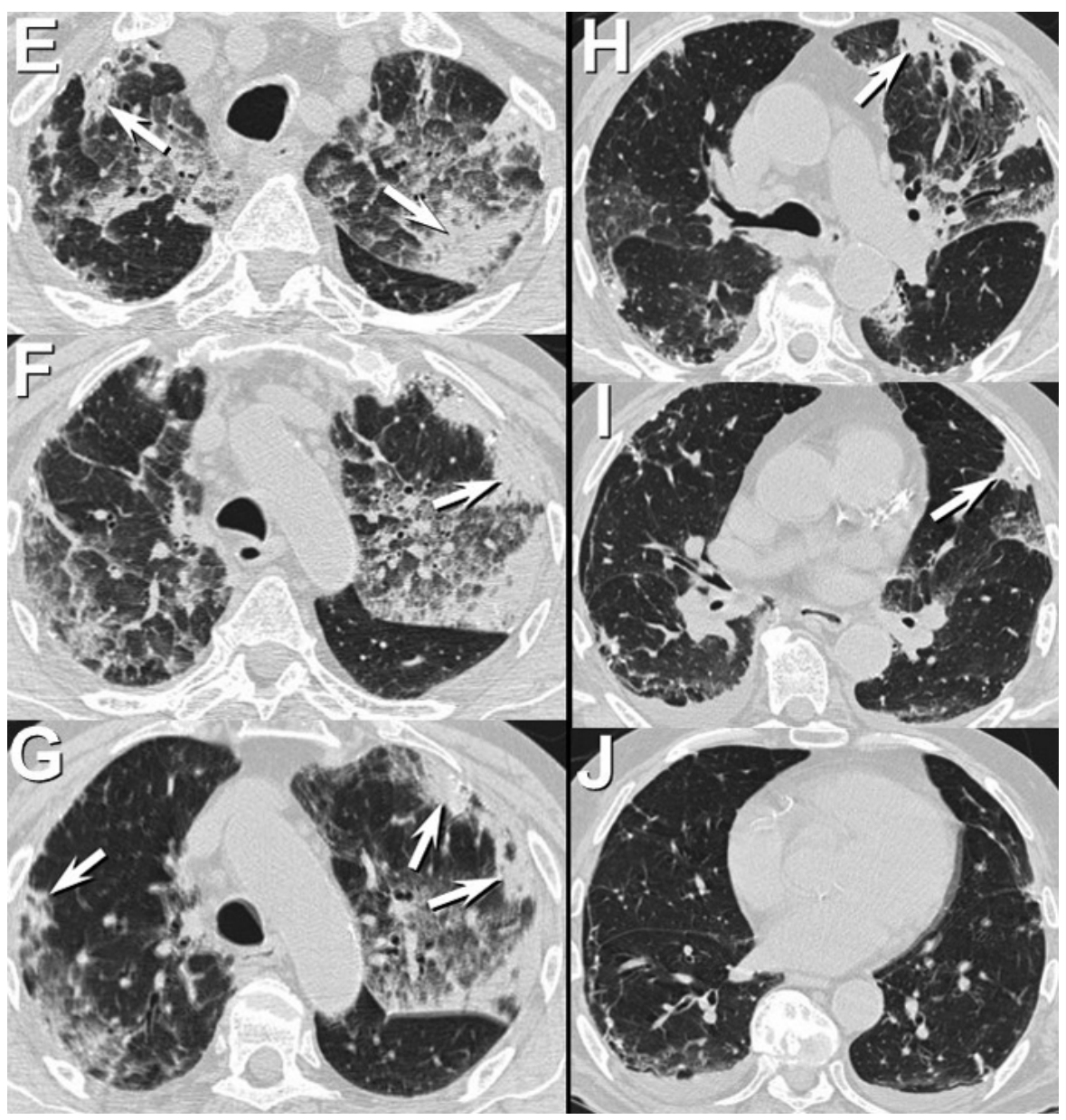

Figure 3. Panels E-J. Selected static images from the axial high-resolution thoracic CT again shows the peripheral and frankly subpleural consolidation (arrows), highlighting the upper lobe distribution.

Central peribronchovascular thickening is not a striking feature on the images provided. The opacities are clearly upper lobe predominant, as shown on the chest radiograph, and not lower lobe predominant. Peripheral endobronchial airway impaction typically presents with small centrilobular nodules, often with a branching configuration, commonly referred to as "tree-in-bud" opacity; such nodules are not present on these images. The images provided do not show multifocal ground-glass opacity nodules. The opacities on the CT study are not cavitary; air bronchograms are present, but cavitary necrosis is lacking.)

What is the appropriate next step for the evaluation / management of this patient?

1. ${ }^{18 F}$ FDG-PET scanning

2. Complete blood count with differential

3. Percutaneous transthoracic biopsy

4. Presumptive antibiotic therapy for fungal infection

5. Surgical lung biopsy 


\section{Correct! \\ 2. Complete blood count with differential}

The imaging findings could be caused by fungal infection, but other, more likely, considerations remain, and none of the data provided thus far directly suggests fungal infection. ${ }^{18 \mathrm{~F}} \mathrm{FDG}-\mathrm{PET}$ scanning would likely not provide management-altering information- elevated tracer uptake in the lung opacities could be the result of inflammation or neoplasm, whereas lack of tracer utilization would not obviate the need for investigation of the abnormal chest radiography and thoracic CT findings. Surgical lung biopsy is not an incorrect choice, given that it is a very useful technique for the evaluation of diffuse lung disease. However, such an invasive approach is premature at this point. Percutaneous transthoracic lung biopsy is generally not the procedure of choice for the investigation of diffuse lung abnormalities. A simple test- a complete blood count with differential- is the best choice among those provided. The results of the complete blood count, in combination with the thoracic CT findings, may provide enough information for a working diagnosis.

Which of the following results of the complete blood count correlate best with the differential diagnostic considerations based on the imaging findings?
1. Anemia
2. Eosinophilia
3. Leukocytosis
4. Lymphocytopenia
5. Thrombocytopenia 


\section{Correct! \\ 2. Eosinophilia}

The differential diagnostic considerations for the imaging findings correlate based with the presence of peripheral eosinophilia. The other choices are certainly possible, but are non-specific in the context of the imaging findings.)

The complete blood count with differential revealed 8\% eosinophilia, with an absolute eosinophil count of $1200 / \mathrm{mm}^{3}$.

What is the appropriate next step for the evaluation / management of this patient?

1. Barium swallow to assess for possible gastroesophageal reflux

2. Bronchoscopy with bronchoalveolar lavage

3. Contrast-enhanced thoracic MRI to assess for vasculitis

4. Presumptive antifungal therapy

5. Sputum analysis for non-tuberculous mycobacteria 


\section{Correct! \\ 2. Bronchoscopy with bronchoalveolar lavage}

The presence of peripheral eosinophilia may be noted in a number of conditions, but, in the context of peripheral lung opacities detected at thoracic imaging, the differential diagnostic considerations narrow to primarily include chronic eosinophilic pneumonia, Churg-Strauss syndrome, pulmonary toxic mediation reactions, and rare disorders associated with eosinophilia, such as parasitic infection, and simple pulmonary eosinophilia (Löffler syndrome). Serological assessment for vasculitis, stool analysis for ova and parasites, and review of medication usage are all appropriate next steps for this patient's management. However, the imaging and laboratory features do not suggest non-tuberculous mycobacterial infection, nor are they highly suggestive of gastroesophageal reflux disease, and therefore assessment for these conditions is not immediately required. Fungal infection remains a possibility, but less likely than other causes of peripheral eosinophilia noted previously, and therefore presumptive antifungal therapy is not indicated. While a vasculitis, particularly Churg-Strauss syndrome, remains possible, thoracic MR assessment for this disorder is premature. The next appropriate step for the management for this patient is bronchoscopy with bronchoalveolar lavage.

The patient subsequently underwent bronchoscopy with bronchoalveolar lavage, which disclosed $40 \%$ eosinophilia in the lavage fluid. Serological assessment for vasculitis and stool analysis for ova and parasites were both unremarkable. The constellation of clinical, laboratory, and imaging features was considered consistent with chronic eosinophilic pneumonia, and corticosteroid therapy was subsequently initiated and the patient rapidly improved over the next few days.

Diagnosis: Chronic eosinophilic pneumonia

\section{References}

1. Alam M and Burki NK. Chronic eosinophilic pneumonia: a review. South Med J. 2007;100(1):49-53. [CrossRef] [PubMed]

2. Jeong YJ, Kim KI, Seo IJ, Lee CH, Lee KN, Kim KN, Kim JS, Kwon WJ. Eosinophilic lung diseases: a clinical, radiologic, and pathologic overview. Radiographics. 2007;27(3):617-637; discussion 637-639. [CrossRef] [PubMed]

3. Arakawa $\mathrm{H}$, Kurihara $\mathrm{Y}$, Niimi $\mathrm{H}$, Nakajima $\mathrm{Y}$, Johkoh T, Nakamura $\mathrm{H}$. Bronchiolitis obliterans with organizing pneumonia versus chronic eosinophilic pneumonia: highresolution CT findings in 81 patients. AJR Am J Roentgenol. 2001;176(4):10531058. [CrossRef] [PubMed]

4. Cottin V and Cordier JF. Eosinophilic lung diseases. Immunol Allergy Clin North Am. 2012;32(4):557-586. [CrossRef] [PubMed] 